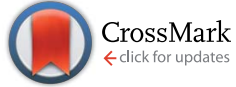

Cite this: RSC Adv., 2014, 4, 56248

\title{
Solid-state NMR measurements and DFT calculations of the magnetic shielding tensors of protons of water trapped in barium chlorate monohydrate
}

\author{
Diego Carnevale, ${ }^{* a}$ Sharon E. Ashbrook ${ }^{\mathrm{b}}$ and Geoffrey Bodenhausen ${ }^{\text {acde }}$
}

Received 8th September 2014

Accepted 15th October 2014

DOI: $10.1039 / \mathrm{c} 4 \mathrm{ra0} 0992 \mathrm{c}$

www.rsc.org/advances

\begin{abstract}
The magnetic shielding tensors of protons of water in barium chlorate monohydrate are investigated at room temperature by means of solid-state NMR spectroscopy, both for static powders and under magic-angle spinning conditions, using one- and two-dimensional techniques. First-principles DFT calculations based on a periodic planewave pseudopotential formalism for a static periodic system provide support for our spectral interpretation and corroborate the experimental findings in the fast motion regime.
\end{abstract}

\section{Introduction}

Water is one of the most abundant molecules on earth and plays a fundamental role in a wide variety of chemical systems, ranging from the biochemical pathways underlying the most complex functions of living systems to the mechanical properties of the Earth's crust. Whether considered as an isolated molecule in the gas phase or in liquid or solid condensed phases, many aspects of water remain not yet properly understood. ${ }^{1-3}$ Hydrogen bonding, chemical exchange and dynamics complicate the description of the system.

Recently, attention has been drawn to the possibility of manipulating the populations of the proton spin eigenstates of water in view of exciting a long-lived state in analogy with para$\mathrm{H}_{2} \cdot{ }^{4} \mathrm{~A}$ knowledge of all nuclear spin interactions which can affect and perturb the eigenstates of protons in water is crucial for the design of experimental strategies aiming at establishing long-lived states in any context, whether in liquid bulk, trapped in a crystal or in a fullerene cage. ${ }^{5-7}$ These interactions, i.e., chemical shieldings, dipolar or quadrupolar couplings, are generally anisotropic and orientation dependent, and may affect NMR spectra to an extent that may render spectral interpretation difficult. ${ }^{8}$ Nevertheless, the inhomogeneous broadenings which arise from such interactions in solids can be

${ }^{a}$ Institut des Sciences et Ingénierie Chimiques (ISIC), Ecole Polytechnique Fédérale de Lausanne (EPFL), CH-1015 Lausanne, Switzerland. E-mail: diego.carnevale@epfl.ch ${ }^{b}$ School of Chemistry, EaStCHEM and Centre of Magnetic Resonance, University of St Andrews, North Haugh, St Andrews, KY16 9ST, UK

'École Normale Supérieure-PSL Research University, Département de Chimie, 24, rue Lhomond, F-75005 Paris, France

${ }^{d}$ Sorbonne Universités, UPMC Univ Paris 06, LBM, 4 place Jussieu, F-75005, Paris, France

${ }^{e}$ CNRS, UMR 7203 LBM, F-75005, Paris, France thoroughly studied by NMR spectroscopy. The use of magicangle spinning (MAS) can partially remove this broadening to yield high-resolution spectra that benefit from a gain in signal intensity. ${ }^{9,10}$ Specific experiments may be used to reintroduce the anisotropic information averaged out by the mechanical rotation. All these capabilities identify solid-state NMR spectroscopy as a method of choice for investigations of the interactions that can affect nuclear spin states. Density Functional Theory (DFT) calculations based on a planewavepseudopotential formalism ${ }^{11,12}$ can nowadays be readily performed for periodic systems made up of a few hundred atoms. Such in silico calculations provide insight into observable properties, such as chemical shielding tensors and electric field gradients, that have proven extremely useful to assist the interpretation of NMR spectra of solid samples. ${ }^{13-15}$

In this context, we turned our attention to the protons of water molecules trapped in crystals of barium chlorate monohydrate, $\mathrm{Ba}\left(\mathrm{ClO}_{3}\right)_{2} \cdot \mathrm{H}_{2} \mathrm{O}$. The anisotropy of the chemical shifts of the protons has been investigated previously by NMR spectroscopy, both in solution and solid state. ${ }^{16,17}$ Here, we further explore the inhomogeneous CSA interaction by refocusing the much larger homonuclear dipolar couplings. The experimental findings are interpreted in the light of the results from DFT calculations.

\section{Results and discussion}

Fig. 1a shows a static proton NMR spectrum of barium chlorate monohydrate, $\mathrm{Ba}\left(\mathrm{ClO}_{3}\right)_{2} \cdot \mathrm{H}_{2} \mathrm{O}$, acquired at room temperature in a $9.4 \mathrm{~T}$ magnet by means of a $90_{y}^{\circ}-\tau-90_{\phi}^{\circ}-\tau$ solid echo experiment. When phase cycling is applied to the second $90^{\circ}$ pulse to select the $p=+1 \rightarrow p=-1$ coherence pathway, this experiment can refocus both linear and quadratic interactions, i.e., both 

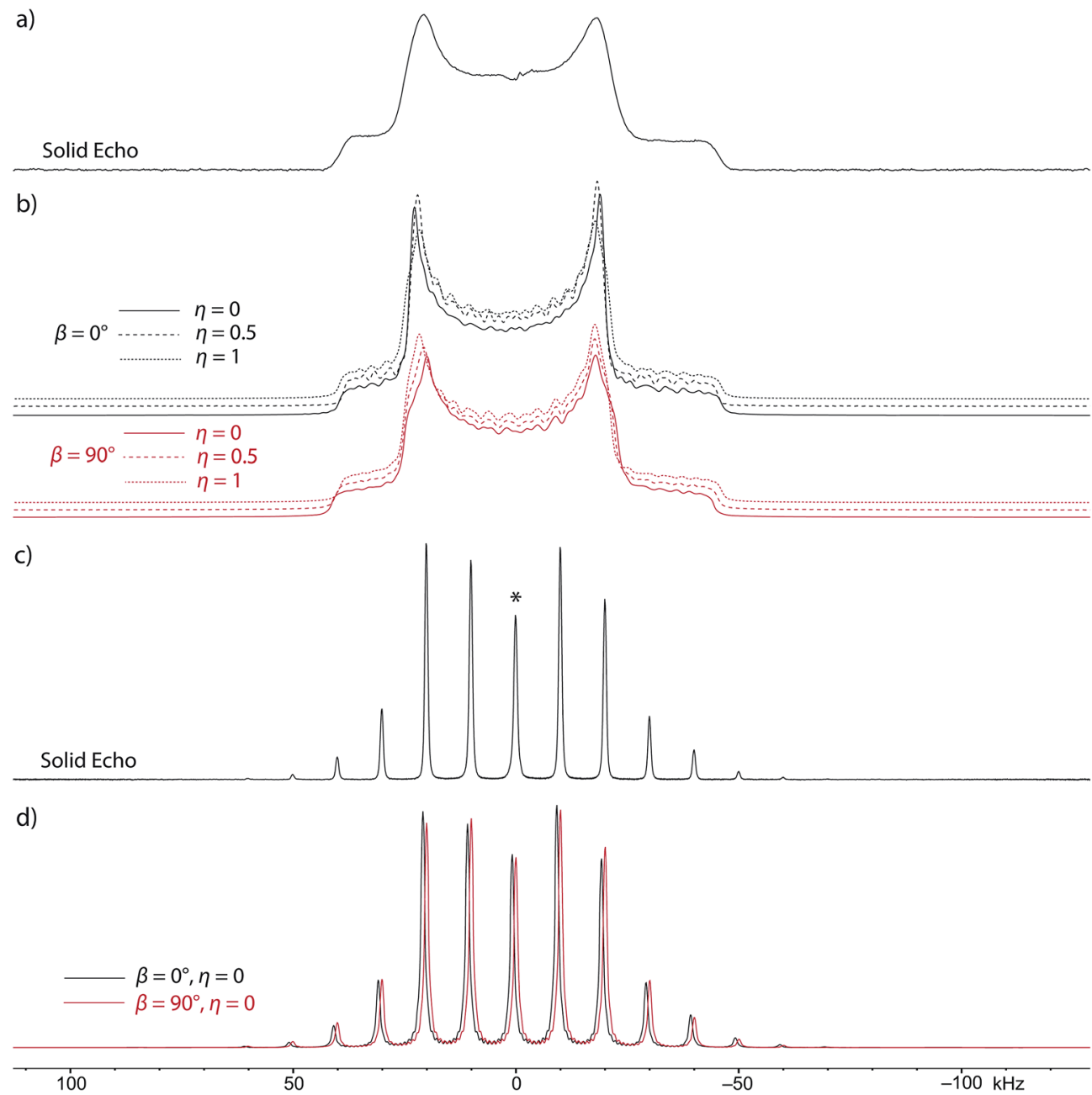

Fig. 1 (a) Experimental ${ }^{1} \mathrm{H}$ NMR spectrum of a static powder of $\mathrm{Ba}\left(\mathrm{ClO}_{3}\right)_{2} \cdot \mathrm{H}_{2} \mathrm{O}$ acquired with a solid echo on a $9.4 \mathrm{~T}$ magnet. (b) Numerical simulations of Pake patterns resulting from two protons subject to a shielding anisotropy $\Delta_{\mathrm{cS}}=4 \mathrm{kHz}, \eta_{\mathrm{cs}}=0,0.5$ and 1.0 , and a dipolar coupling constant $d=-29 \mathrm{kHz}$, with relative orientations of the two tensors $\beta=0$ and $90^{\circ}$, in black and red, respectively. (c) Experimental ${ }^{1} \mathrm{H}$ NMR spectrum acquired with a rotor-synchronized solid echo on a $9.4 \mathrm{~T}$ magnet at $\nu_{\text {rot }}=10 \mathrm{kHz}$. (d) Simulations of the spinning sideband pattern of (c) when $\beta=0$ and $90^{\circ}$, in black and red, respectively. In all cases, the carrier frequency was set to coincide with the isotropic peak $(*), i . e ., \nu_{\text {rf }}=\delta_{\text {iso }}=$ $1.68 \mathrm{kHz}=4.2 \mathrm{ppm}$.

shift anisotropies and dipolar couplings. ${ }^{18}$ The features of the lineshape clearly indicate what is commonly referred to as a Pake pattern due to isolated homonuclear dipolar-coupled spin pairs, originally observed in gypsum that, in contrast with our system, occurs as a dihydrate, i.e., $\mathrm{CaSO}_{4} \cdot 2 \mathrm{H}_{2} \mathrm{O} .{ }^{19}$ The mere observation of such a powder pattern indicates that the water molecules are sufficiently remote from each other in the crystal that intermolecular couplings between protons of different water molecules do not contribute significantly to the lineshape. It is interesting to note the asymmetry of the powder pattern, which was not observed in Pake's pioneering study on gypsum, presumably because he worked at a much lower field of $0.682 \mathrm{~T}$. It can easily be appreciated that the low-frequency (right-hand) shoulder of the pattern in Fig. 1a is broader than its high-frequency counterpart. This asymmetry has been already observed in spinning powders and correctly ascribed to the chemical shift anisotropy of the proton spins. ${ }^{17}$ Fig. $1 \mathrm{~b}$ shows a series of numerical simulations that include a chemical shift anisotropy $\Delta_{\mathrm{CS}}$ reproduce well the asymmetry of the experimental powder pattern in Fig. 1a. However, as indicated by the series of spectra stacked in Fig. 1b for different asymmetry parameters of the shielding tensor $\eta_{\mathrm{CS}}=0,0.5$ and 1 , the effect on the lineshape is less pronounced, with only the 'horns' of the pattern significantly affected. When compared to the experimental spectrum of Fig. 1a, it is clear that even a slight homogeneous broadening can easily hide the subtle changes in the lineshape that result from a variation in $\eta_{\mathrm{CS}}$. All this means that an accurate determination of the asymmetry, $\eta_{\mathrm{CS}}$, form the analysis of 1D static NMR spectra may be, at least in the case at hand, very difficult.

One additional parameter that needs to be taken into account in order to analyze the spectrum in Fig. 1a is the relative orientation of the shielding and dipolar tensors of the proton spins. The latter is aligned along the $\mathrm{H}-\mathrm{H}$ vector whereas the 
former, when they are axially symmetric, i.e., in the limit where $\eta_{\mathrm{CS}}=0$, are usually assumed to have their unique axis aligned parallel to their respective $\mathrm{H}-\mathrm{O}$ bonds. In solid-state systems, when dealing with more than one interaction, three Euler angles $\Omega=(\alpha, \beta, \gamma)$ are required to describe their relative orientations. Each interaction can be defined in its own principal axis frame (P), where the relevant tensor is diagonal. A crystal frame (C) may also be considered so that all interactions can be referred to a common frame of reference. Consequently, each interaction $\lambda$ has a specific set of Euler angles $\Omega_{\mathrm{PC}}^{\lambda}=$ $\left(\alpha_{\mathrm{PC}}, \beta_{\mathrm{PC}}, \gamma_{\mathrm{PC}}\right)$. Consideration of the rotor frame $(\mathrm{R})$ is also required for MAS experiments. Finally, the lab frame $(\mathrm{L})$, where the experiment takes place, is also needed. It is legitimate, and adopted in this study, to assume the $P$ frame of a given interaction $\lambda$ to be coincident with the common $\mathrm{C}$ frame. This is simply done by choosing $\Omega_{\mathrm{PC}}^{\lambda}=\left(0^{\circ}, 0^{\circ}, 0^{\circ}\right)$. In our case, for the two-spin system of a single isolated water molecule, two shielding tensors and one dipolar tensor need be taken into account.

However, water molecules that are trapped in solids are known to undergo rapid reorientation by flipping around the $C_{2}$ axis defined by the $\mathrm{H}-\mathrm{O}-\mathrm{H}$ bisector. ${ }^{20}$ In the fast motional regime at room temperature, it is commonly assumed that such motions result in an average shielding tensor projected onto the $C_{2}$ axis, so that its main axis is, therefore, orthogonal to that of the dipolar tensor. As a result, the shielding tensors of the two protons are equivalent and collinear at room temperature. In contrast, a rotation about the $C_{2}$ axis has no effect on the dipolar tensor since a $180^{\circ}$ flip does not alter the size of this interaction. Therefore, in order to simulate the lineshapes, we assume the spin system to be made up of two $I=1 / 2$ spins, with equivalent shielding tensors that are collinear, and with two $P$ frames that are coincident with the common $\mathrm{C}$ frame. Consequently, we have three sets of Euler angles, ${ }^{\mathrm{H}(1)} \Omega_{\mathrm{PC}}^{\mathrm{CS}}=\left(0^{\circ}, 0^{\circ}, 0^{\circ}\right)={ }^{\mathrm{H}(2)} \Omega_{\mathrm{PC}}^{\mathrm{CS}}$ and $\Omega_{\mathrm{PC}}^{\mathrm{D}}$ $=\left(0^{\circ}, \beta_{\mathrm{PC}}, \gamma_{\mathrm{PC}}\right)$. As the dipolar tensor is axially symmetric and traceless, only two angles, say, $\beta_{\mathrm{PC}}$ and $\gamma_{\mathrm{PC}}$ (henceforth simply referred to as $\beta$ and $\gamma$ ) are relevant, i.e., $\alpha_{\mathrm{PC}}$ is redundant and assumed to be $0^{\circ}$ in this context. ${ }^{21}$ In Fig. $1 \mathrm{~b}$, static patterns are simulated for two cases of $\Omega_{\mathrm{PC}}^{\mathrm{D}}=\left(0^{\circ}, 0^{\circ}, 0^{\circ}\right)$ and $\left(0^{\circ}, 90^{\circ}, 0^{\circ}\right)$, in black and red, respectively. As previously discussed for the asymmetry parameter $\eta_{\mathrm{CS}}$ of the shielding tensor, the angle $\beta$ has very little effect on the static lineshape. If a systematic fit of the spectrum of Fig. 1a is performed over the two-dimensional space spanned by the parameters $\Delta_{\mathrm{CS}}$ and $\eta_{\mathrm{CS}}$, for the case of $\beta=90^{\circ}$, one finds $\Delta_{\mathrm{CS}}=11 \pm 3 \mathrm{ppm}$ and $\eta_{\mathrm{CS}}=0.3 \pm 0.5$. Clearly, the error associated with the asymmetry is too large to be reliable. An analogous fit for the case $\beta=0^{\circ}$ produces substantially identical parameters, i.e., $\Delta_{\mathrm{CS}}=-10 \pm 3 \mathrm{ppm}$ and $\eta_{\mathrm{CS}}=1.0 \pm 0.7$, meaning once more that $\beta$ cannot be determined. It is worth noting that a fit assuming $\Omega_{\mathrm{PC}}^{\mathrm{D}}=\left(0^{\circ}, 90^{\circ}, 0^{\circ}\right)$ results in $\Delta_{\mathrm{CS}}>$ 0 whereas the case of $\Omega_{\mathrm{PC}}^{\mathrm{D}}=\left(0^{\circ}, 0^{\circ}, 0^{\circ}\right)$ produces $\Delta_{\mathrm{CS}}<0$.

Fig. 1c shows a magic-angle spinning (MAS) spectrum recorded at 9.4 $\mathrm{T}$ with a rotor-synchronized solid echo using a spinning frequency of $\nu_{\text {rot }}=10 \mathrm{kHz}$. The intensities of the spinning sidebands are markedly asymmetric with respect to the isotropic shift (marked by ${ }^{*}$ ), which, in analogy with the static case, can be ascribed to the chemical shift anisotropy. ${ }^{17}$
Fig. 1d shows two simulations for the two cases of $\beta=0$ and $90^{\circ}$, in black and red, respectively. The black spectrum is slightly shifted to higher frequencies for clarity. The two spectra are again remarkably similar, revealing only tiny differences in the intensities of the spinning sidebands. Only the cases of $\eta_{\mathrm{CS}}=$ 0 are shown, since variations of this parameter produce even smaller effects than variations of $\beta$. Attempts to fit the spectrum of Fig. 1c result in very large uncertainties of the relevant parameters, reflecting the fact that they have little effect on the lineshape. More specifically, one obtains $\Delta_{\mathrm{CS}}=-9 \pm 7 \mathrm{ppm}$ and $\eta_{\mathrm{CS}}=0 \pm 11$ in the case of $\Omega_{\mathrm{PC}}^{\mathrm{D}}=\left(0^{\circ}, 0^{\circ}, 0^{\circ}\right)$ and $\Delta_{\mathrm{CS}}=9 \pm 10$ ppm and $\eta_{\mathrm{CS}}=1 \pm 2$ in the case of $\Omega_{\mathrm{PC}}^{\mathrm{D}}=\left(0^{\circ}, 90^{\circ}, 0^{\circ}\right)$. As previously observed for the fits of Fig. $1 \mathrm{~b}$, the cases of $\beta=0$ and $90^{\circ}$ yield, respectively, negative and positive values for the shift anisotropy $\Delta_{\mathrm{CS}}$. The lack of both accuracy and precision which affects these measurements can be rationalized by considering that the inhomogeneity due to the shielding interaction is almost completely averaged by MAS, since $\Delta_{\mathrm{CS}} \approx 4 \mathrm{kHz}$ and $\nu_{\text {rot }}$ $=10 \mathrm{kHz}$. Therefore, an accurate measurement of the shielding tensor with one-dimensional NMR techniques seems to be difficult under both static and MAS conditions in this case, where the size of the predominant dipolar interaction, i.e., ca. $30 \mathrm{kHz}$, and homogeneous broadening mask the effects of the shielding anisotropy.

In order to gain insight into the system under investigation, and to corroborate and interpret the inhomogeneities that were measured experimentally, periodic planewave pseudopotential DFT calculations were carried out with the CASTEP $\operatorname{code}^{22}$ on the periodic system. Fig. 2a-c show the unit cell of $\mathrm{Ba}\left(\mathrm{ClO}_{3}\right)_{2} \cdot \mathrm{H}_{2} \mathrm{O}$ viewed down the $x$-, $y$ - and $z$-axes, respectively. The unit-cell lengths are $a=8.92 \AA, b=7.83 \AA$ and $c=9.43 \AA$, and the angle $\beta=93.39^{\circ} .{ }^{23}$ The space group is $C 2 / c$. Four water mole-

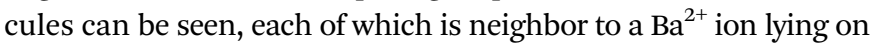
its $C_{2}$ axis. When the magnetic shielding tensors of protons in $\mathrm{Ba}\left(\mathrm{ClO}_{3}\right)_{2} \cdot \mathrm{H}_{2} \mathrm{O}$ are computed before geometry optimization, all protons of all water molecules are characterized by the same main components of their shielding tensors. In contrast, if geometry optimization is performed, this degeneracy is broken and two types of water can be identified. Nevertheless, the proton sites within each water molecule are always identical to one another. If the unit cell size is fixed and conservation of the symmetry is imposed in the geometry optimization step, the differences between the two types of water tend to disappear. The latter condition has a smaller effect on the calculated shielding tensors than the former. Fig. $2 d$ shows the magnetic shielding tensors of the proton sites represented as light-brown ellipsoids. Once expressed in their principal axis frames, all protons are characterized by the same main components of the shielding tensor. The relative orientation between the two tensors of each water molecule is described by the Euler angles $\left(92.83^{\circ}, 65.47^{\circ}, 92.83^{\circ}\right)$. The relevant NMR parameters obtained are summarized in Table 1 . Generally, DFT calculations yield $\Delta_{\mathrm{CS}}=-16.5 \mathrm{ppm}$ and $\eta_{\mathrm{CS}}=0.2$. Note that very little difference is obtained between different methods for structural optimization. The computational investigation is performed on a static system, so that motional averaging of the interactions is not taken into account. As we expect the two tensors to be averaged 
a)

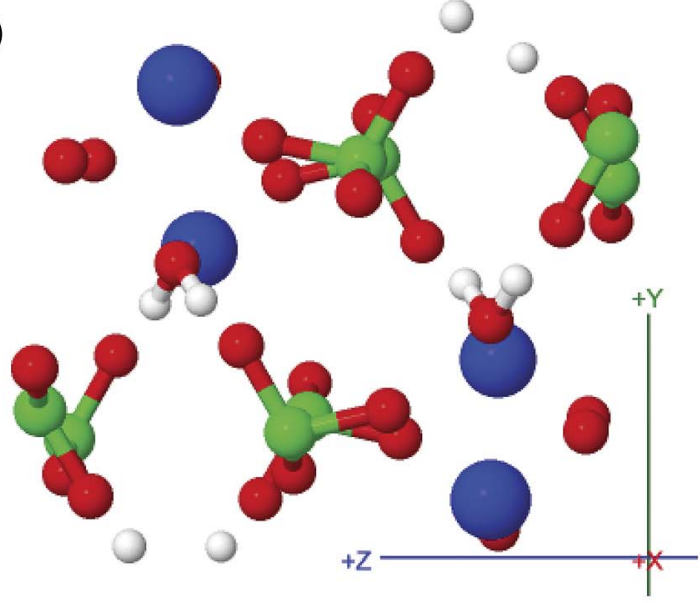

c)

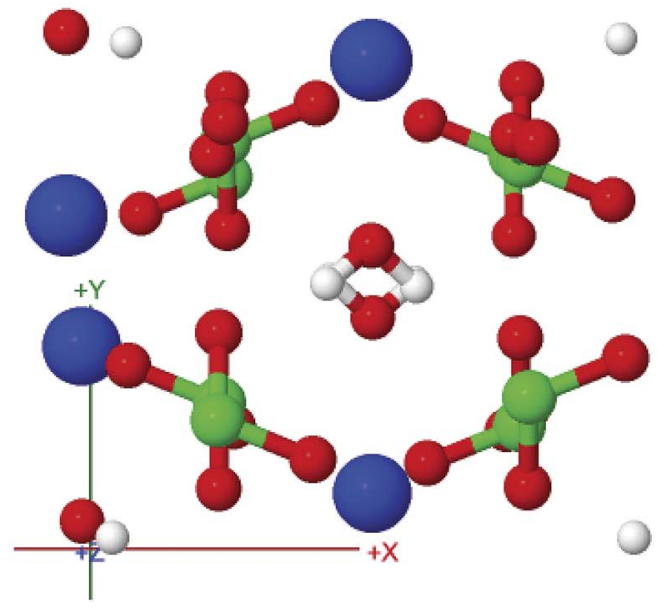

b)

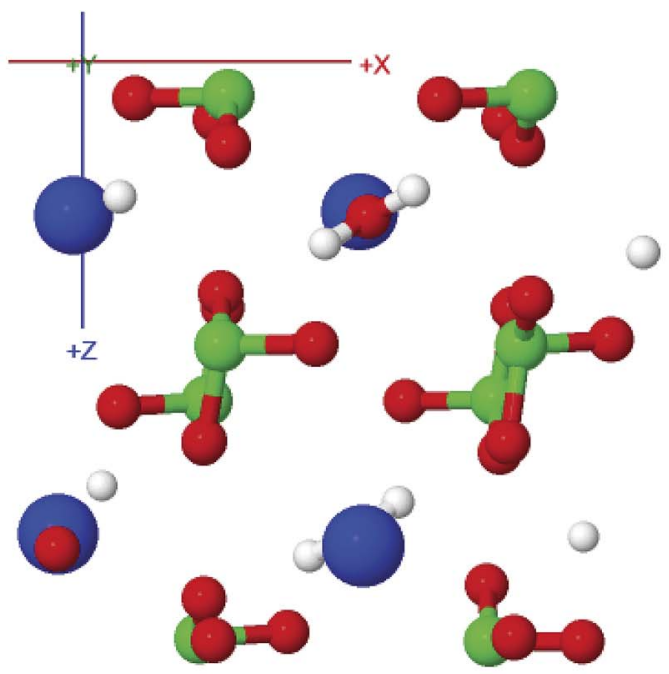

d)

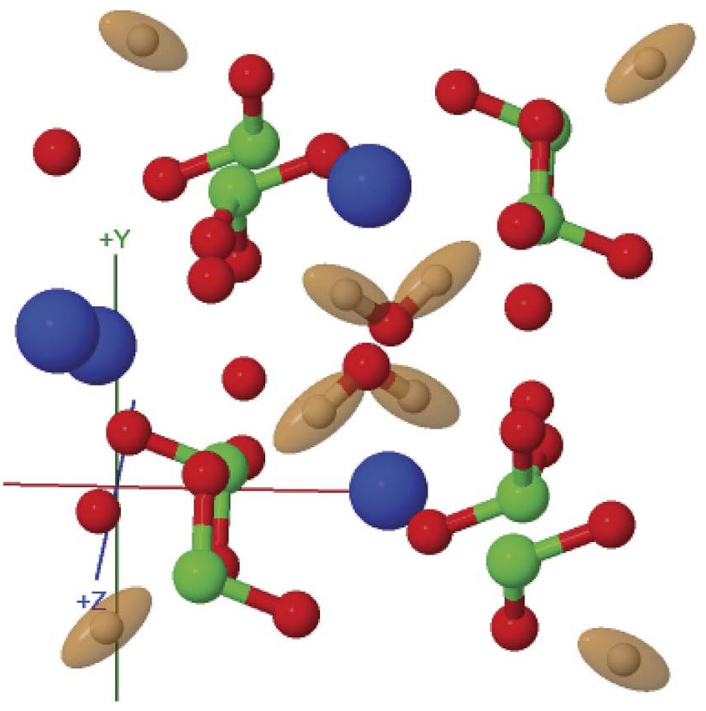

Fig. 2 Unit cell of $\mathrm{Ba}\left(\mathrm{ClO}_{3}\right)_{2} \cdot \mathrm{H}_{2} \mathrm{O}$ viewed down the $x-, y$ - and $z$-axes is shown in (a), (b) and (c), respectively. Oxygen, proton, barium and chlorine atoms are shown in red, white, blue and green, respectively. (d) Representation of the magnetic shielding tensors of proton nuclei as light-brown ellipsoids.

by fast dynamics at room temperature, we express the two shielding tensors of the two protons $\mathrm{H}(1)$ and $\mathrm{H}(2)$ belonging to a single water molecule in a common frame by means of the following transformations:

$$
\sigma_{\mathrm{H}(1)}^{\prime}=R^{-1}(\alpha, \beta, \gamma) \sigma_{\mathrm{H}(1)} R(\alpha, \beta, \gamma),
$$

where the operator $R(\alpha, \beta, \gamma)$ can be decomposed into its constituent rotations:

$$
R(\alpha, \beta, \gamma)=R_{z}(\alpha) R_{y}(\beta) R_{z}(\gamma)
$$

A single rotation operator, say, $R_{z}(\alpha)$, performs a rotation of the shielding tensor of $\mathrm{H}(1), \sigma_{\mathrm{H}(1)}$, through an angle $\alpha$ around the $z$-axis. This produces $\sigma_{\mathrm{H}(1)}^{\prime}$, i.e., $\sigma_{\mathrm{H}(1)}$ expressed in the principal axis of $\sigma_{\mathrm{H}(2)}$. The average tensor $\bar{\sigma}_{\mathrm{H}(1,2)}$ is then simply given by:

$$
\bar{\sigma}_{\mathrm{H}(1,2)}=\left(\sigma_{\mathrm{H}(1)}^{\prime}+\sigma_{\mathrm{H}(2)}\right) / 2,
$$

By diagonalizing $\bar{\sigma}_{\mathrm{H}(1,2)}$ one obtains the principal components of the averaged interaction tensor. The relevant NMR parameters under investigation thus produced are $\bar{\Delta}_{\mathrm{CS}}=-8.7$ ppm and $\bar{\eta}_{\mathrm{CS}}=0.9$. It is worth noticing that the chemical shift anisotropy calculated with DFT methods is negative. These parameters represent the averaged shielding tensor of an averaged ${ }^{1} \mathrm{H}$ site that one can measure in barium chlorate monohydrate in the fast motional regime. If one considers instantaneous jumps of the protons between their two orientations, the details of the dynamic process are irrelevant, so long as the relative orientation between the initial and final configurations is known. The size of the calculated average anisotropy 
Table 1 Reduced chemical shift anisotropy $\Delta_{\mathrm{CS}}=\delta_{z z}-\delta_{\text {iso, }}$, with isotropic shift $\delta_{\text {iso }}=\left(\delta_{x x}+\delta_{y y}+\delta_{z z}\right) / 3$, and asymmetry $\eta_{C S}=\left(\delta_{x x}-\delta_{y y}\right) /$ $\Delta_{\mathrm{CS}}$ for the two proton sites in barium chlorate monohydrate as calculated with DFT methods implemented in CASTEP in a periodic planewave-pseudopotential formalism. Calculations have been performed without and with geometry optimization (Opt). In this latter case the crystallographic degeneracy of two of the four water molecules is lifted. The possibilities of fixing the unit-cell size (Fix) and imposing conservation of symmetry (Sym) have also been considered

\begin{tabular}{lcllll}
\hline & \multicolumn{2}{l}{ Site 1} & & \multicolumn{2}{l}{ Site 2} \\
\cline { 2 - 3 } \cline { 5 - 6 } \cline { 5 - 6 } & $\Delta_{\mathrm{CS}}(\mathrm{ppm})$ & $\eta_{\mathrm{CS}}(\mathrm{ppm})$ & & $\Delta_{\mathrm{CS}}(\mathrm{ppm})$ & $\eta_{\mathrm{CS}}$ \\
\hline No Opt & -16.69 & 0.15 & & - & - \\
Opt & -16.27 & 0.17 & & -16.19 & 0.17 \\
Opt/Fix & -16.47 & 0.17 & & -16.48 & 0.17 \\
Opt/Sym & -16.29 & 0.17 & & -16.20 & 0.17 \\
Opt/Fix/Sym & -16.45 & 0.17 & & -16.45 & 0.17 \\
Motionally averaged & -8.7 & 0.9 & - & - \\
\hline
\end{tabular}

$\bar{\Delta}_{\mathrm{CS}}$ is in good agreement with the experimental 1D spectra. On the other hand, the uncertainty associated with our measurements of the asymmetry $\eta_{\mathrm{CS}}$ does not allow any reasonable comparison with the averaged value calculated with DFT methods.

The optimal method for the measurement of the shielding tensors of protons in $\mathrm{Ba}\left(\mathrm{ClO}_{3}\right)_{2} \cdot \mathrm{H}_{2} \mathrm{O}$ would be a twodimensional technique capable of isolating the shift interaction in the indirect dimension, producing a pure-shift $F_{1}$ projection where the predominant dipolar interaction has been removed by refocusing. Antonijevic and Wimperis have proposed a two-dimensional NMR method to refocus the firstorder quadrupolar interaction of deuterium spins $(I=1)$ in the indirect dimension of a static $2 \mathrm{D}$ spectrum. ${ }^{21}$ The basic principle of this experiment relies on a solid echo in the center of a $t_{1}$ evolution to refocus the quadratic or bilinear Hamiltonians such as dipolar or first-order quadrupolar couplings, whereas modulations due to linear terms such as that of the inhomogeneous Zeeman Hamiltonian are retained. This can be achieved if the second $90^{\circ}$ pulse is phase cycled to select the $p=$ $+1 \rightarrow p=+1$ coherence pathway. ${ }^{21}$ A pure-shift $F_{1}$ dimension is thus achieved. Although specifically designed for ${ }^{2} \mathrm{H}$ spins $(I=$ 1), it is easy to verify that the same result can be achieved for a $I$ $=1 / 2$ spin pair with equivalent shielding tensors subject to a homonuclear dipolar coupling. This condition applies in our case of two equivalent tensors whose different orientations are averaged in the fast motional regime at room temperature. Although the space parts are different, the first-order quadrupolar interaction and homonuclear dipolar interaction have the same bilinear spin parts, i.e., $T=3 I_{z} S_{z}-I S$, where $I=S$ if $I=$ 1. The evolution during the $t_{1}$ interval of the $90_{y}^{\circ}-t_{1} / 2-90_{x}^{\circ}-t_{1} / 2$ experiment may be represented by the following transformations:

$$
\rho_{1}=U_{\mathrm{CS}}\left(t_{1} / 2\right) U_{\mathrm{D}}\left(t_{1} / 2\right) \rho_{0} U_{\mathrm{D}}^{\dagger}\left(t_{1} / 2\right) U_{\mathrm{CS}}^{\dagger}\left(t_{1} / 2\right),
$$

$$
\begin{gathered}
\rho_{2}=U_{\mathrm{rr}}\left(90_{x}^{\circ}\right) \rho_{1} U_{\mathrm{rf}}^{\dagger}\left(90_{x}^{\circ}\right), \\
\rho_{3}=U_{\mathrm{CS}}\left(t_{1} / 2\right) U_{\mathrm{D}}\left(t_{1} / 2\right) \rho_{2} U_{\mathrm{D}}^{\dagger}\left(t_{1} / 2\right) U_{\mathrm{CS}}^{\dagger}\left(t_{1} / 2\right),
\end{gathered}
$$

where $\rho_{0}=I_{+}+S_{+}$for a system with two spins $I=S=1 / 2$ or $I_{+}$ for a single $I=1$ spin. Ideal pulses are considered and the order in which shift and dipolar (or first-order quadrupolar) interactions are treated is irrelevant, as the corresponding Hamiltonians commute with one another. In the case of two $I$ $=1 / 2$ spins, the final state $\rho_{3}$ is characterized by singlequantum matrix elements $\rho_{3}^{(p=+1)}$ which are modulated exclusively by the shift interaction, i.e., $\operatorname{Tr}\left[I_{-} \rho_{3}\right]=2 \exp \left(-i 2 \tau \omega_{\mathrm{CSA}}\right)$. It is worth noting that the single-quantum matrix elements $\rho_{3}^{(p=-1)}$ are instead completely unmodulated, i.e., $\operatorname{Tr}\left[I_{+} \rho_{3}\right]=2$. This latter feature is utilized in the experiments of Fig. 1. Fig. 3a shows a two-dimensional NMR spectrum of a static powdered sample of barium chlorate monohydrate. The $F_{2}$ dimension is, of course, affected by both CSA and dipolar interactions, whereas pure-shift information appears in $F_{1}$. It is worth noting that the horizontal $F_{2}$ projection appears slightly distorted when compared to the static spectrum acquired with a solid echo and shown in Fig. 1a. This is due to the fact that a whole echo ${ }^{24}$ has been acquired in the $2 \mathrm{D}$ experiment whereas, in the case of the 1D spectrum, acquisition is started at the very top of the echo. In the former case, but not in the latter, unavoidable homogeneous losses act 'asymmetrically' around the top of the echo. Moreover, and ideally, an equal number of data points on each side of the top of the echo would be needed for undistorted lineshapes to be obtained. All these deviations from an ideal whole echo result in the distortion of the Pake pattern as observed in the projection of the 2D spectrum of Fig. 3a. Fig. 3b shows the projection of the $2 \mathrm{D}$ spectrum onto the $F_{1}$ axis with corresponding fit, in black and red, respectively. Despite its strength, the dipolar interaction does not affect the lineshape and the shielding tensor is much easier to access. It is important to stress at this point that the pure-shift $F_{1}$ dimension thus produced is independent of the relative orientation of the dipolar and shielding tensors. Furthermore, due to the equivalence of the principal components and orientations of the two proton tensors as a result of fast motional averaging at room temperature, the numerical fit in Fig. $3 \mathrm{~b}$ could be performed by assuming $a$ single $I=1 / 2$ spin species. All these simplifications result in a considerable reduction of the number of variables to consider. Moreover, there is no need for fitting the whole two-dimensional spectrum. Fig. 3c shows the rms deviations associated with the above-mentioned numerical fit as a function of the shift anisotropy $\Delta_{\mathrm{CS}}$ and asymmetry $\eta_{\mathrm{CS}} \cdot{ }^{13}$ The surface shows clear minima that correspond to the optimal parameters, thus indicating a more accurate and precise measurement of the principal components than those obtained from the 1D spectra of Fig. 1. The relevant parameters that we obtain are $\Delta_{\mathrm{CS}}=-10.5 \pm 0.5 \mathrm{ppm}$ and $\eta_{\mathrm{CS}}=0.7 \pm 0.2$, with errors that are considerably smaller than those obtained by the fits of the spectra in Fig. 1. Most importantly, these values agree 
a)

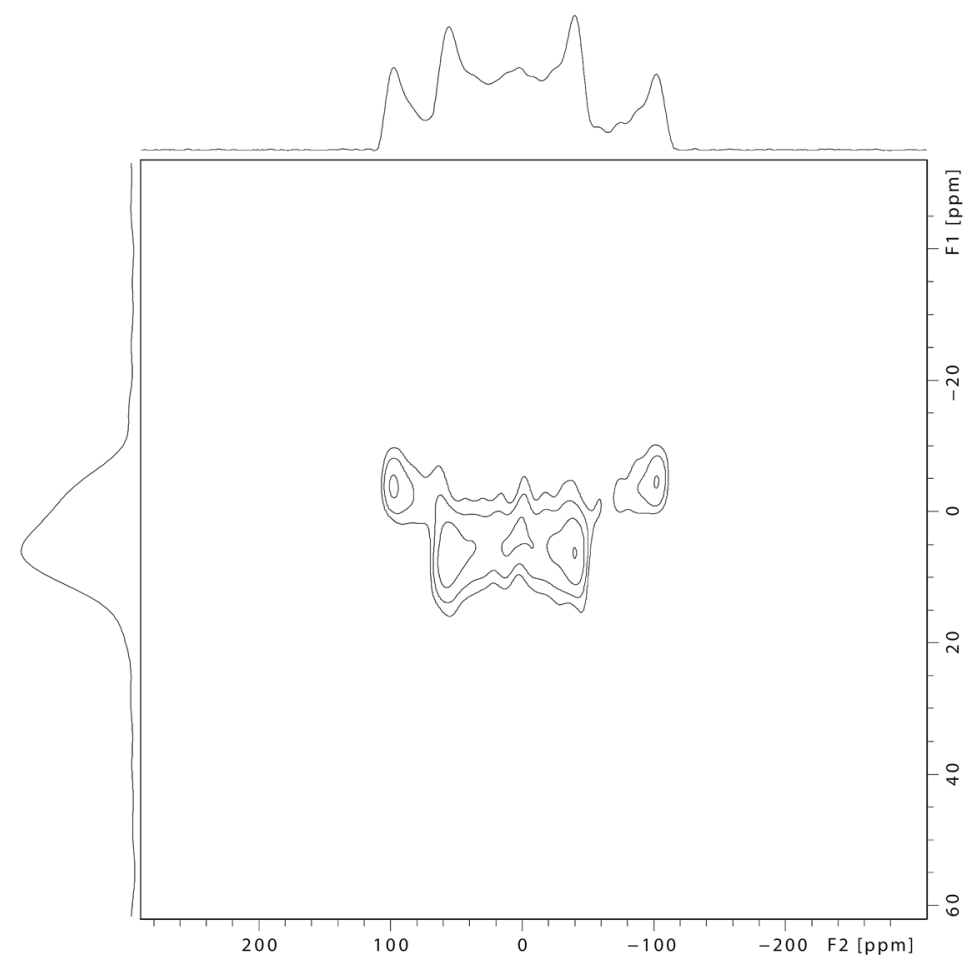

b)

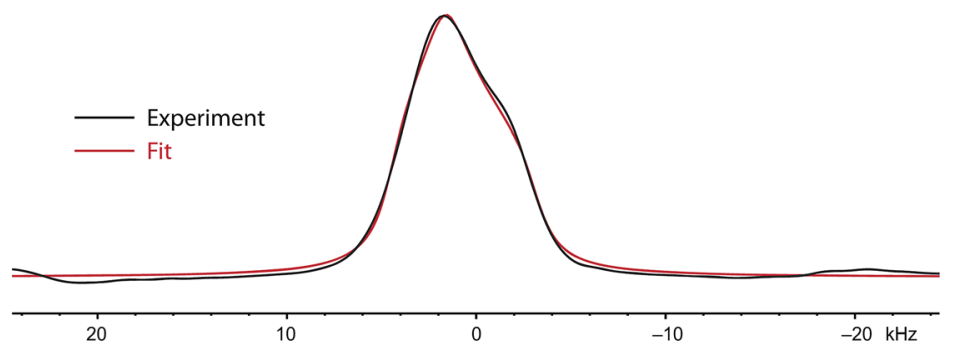

c)

$\square 0-2.5 \quad \square 2.5-5 \quad \square 5-7.5 \quad \square 7.5-10 \quad \square 10-12.5 \square 12.5-15 \backsim 15-17.5 \square 17.5-20 \backsim 20-22.5 \square 22.5-25$

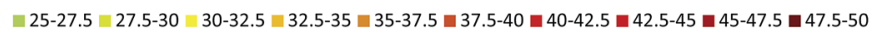

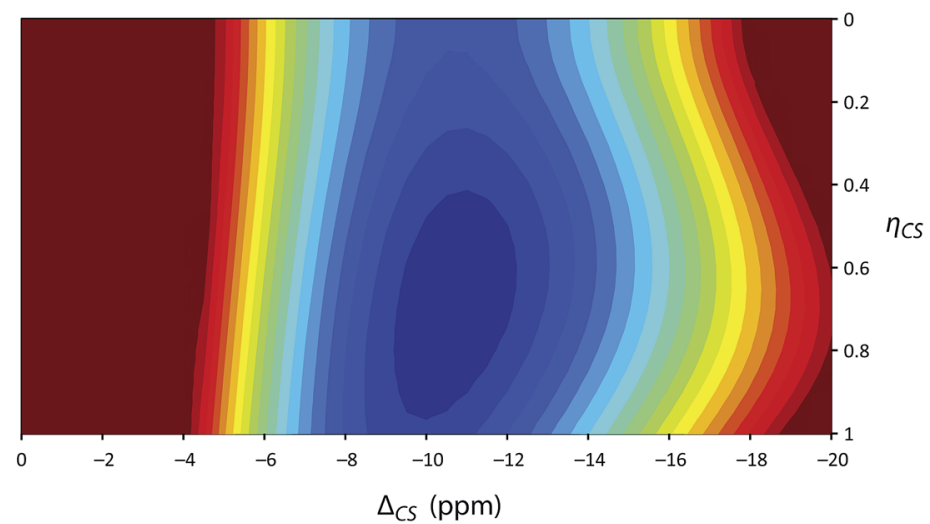

Fig. 3 (a) Experimental two-dimensional correlation between a dipole-shift dimension (horizontally in $F_{2}$ ) and a pure-shift dimension (vertically in $F_{1}$ ) of protons in barium chlorate monohydrate as obtained with the experiment proposed by Antonijevic and Wimperis at 9.4 T. $^{21}$ (b) $F_{1}$ projection of the spectrum (black) in (a) with a fitted simulation (red). (c) Two-dimensional contour plot showing the rms resulting from a systematic fit of the $F_{1}$ projection of the 2D spectrum in (a) over the subspace spanned by the parameters $\Delta_{\mathrm{Cs}}$ and $\eta_{\mathrm{Cs}}$. The intensity scale has been arbitrarily limited to 50 . 
a)

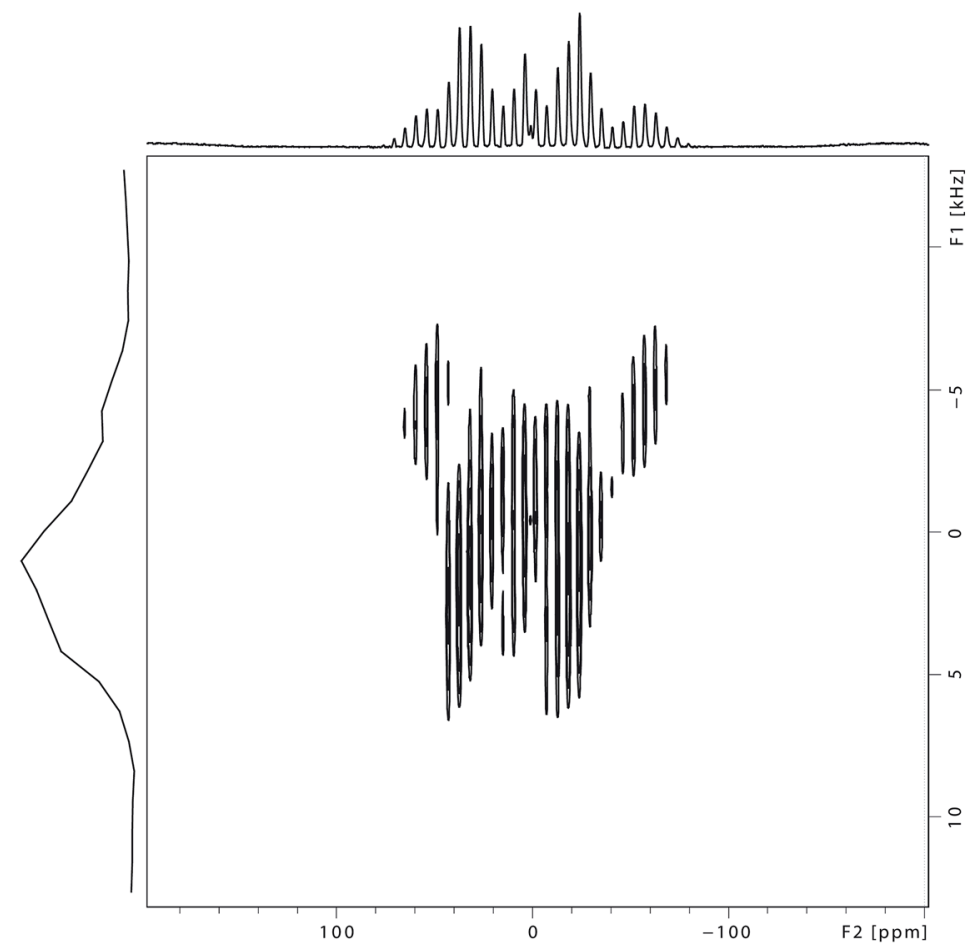

b)

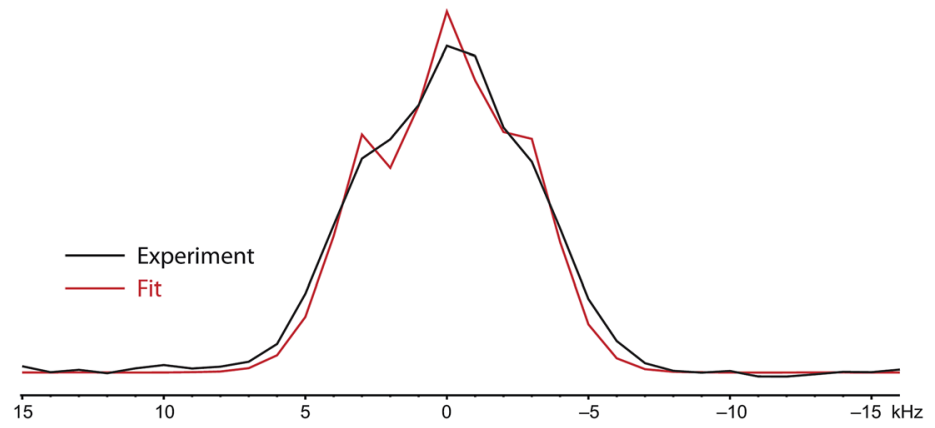

c)

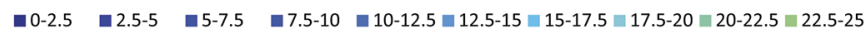
$\varpi 25-27.5 \backsim 27.5-30 \backsim 30-32.5 \backsim 32.5-35 \backsim 35-37.5 \backsim 37.5-40 \backsim 40-42.5 \square 42.5-45 \square 45-47.5 \square 47.5-50$

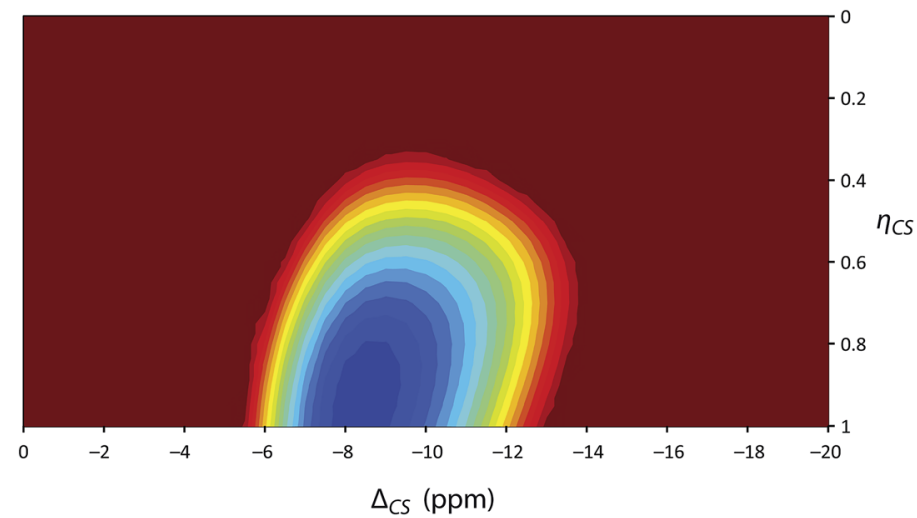

Fig. 4 (a) Experimental two-dimensional correlation between a dipole and shift dimension $\left(F_{2}\right)$ and an amplified pure-shift dimension $\left(F_{1}\right)$ of protons in barium chlorate monohydrate as obtained with the CSA-amplified PASS experiment at 14.1 T. ${ }^{25}$ (b) $F_{1}$ projection of the spectrum (black) in (a) with fit (red). (c) Two-dimensional contour plot showing the $r m s$ resulting from a systematic fit of the $F_{1}$ projection of the 2D spectrum in (a) over the subspace spanned by the $\Delta_{\mathrm{CS}}$ and $\eta_{\mathrm{CS}}$ parameters. The intensity scale has been arbitrarily limited to 50 . 
remarkably well with the averaged parameters calculated with DFT methods.

In cases where the shift anisotropy is small and can easily be removed by MAS, Orr et al. ${ }^{25,26}$ have developed a twodimensional method capable of amplifying the chemical shift anisotropy in the indirect dimension of a twodimensional spectrum. This allows one to have simultaneously the high resolution typical of MAS spectra, and the anisotropic information otherwise averaged out by the mechanical rotation. This experiment is formally equivalent to that of Crockford et al..$^{27,28}$ and has been directly derived as an amplified version of the method of Antzutkin et al. ${ }^{29}$ who revisited the Phase-Adjusted Spinning Sidebands (PASS) ${ }^{30,31}$ experiment with a rigorous formalism. The effect of homonuclear dipolar interactions has been investigated and proven to be deleterious for the desired recoupling and amplification of the chemical shift anisotropy. ${ }^{26}$ However, it can be shown that, in the case of two equivalent spins, the presence of a homonuclear dipolar interaction does not affect the experiment because the dipolar Hamiltonian is not affected by the series of $\pi$ pulses which aim to recouple and amplify the shielding anisotropy. This pulse sequence has been designed by means of first-order Average Hamiltonian Theory (AHT).$^{32}$ Therefore, the total Hamiltonian $H_{\text {Tot }}$ for the relevant spin system needs to commute with itself at different times $t$ and $t^{\prime}$. If this condition is met, higher-order terms of the Magnus expansion $^{33}$ may be discarded and the first-order terms suffice to describe the evolution of the system. In our case, $H_{\mathrm{Tot}}=H_{\mathrm{CS}}^{(l)}$ $+H_{\mathrm{CS}}^{(S)}+H_{\mathrm{D}}$, hence the relevant commutator has the form:

$$
\begin{aligned}
{\left[H_{\mathrm{Tot}}(t), H_{\mathrm{Tot}}\left(t^{\prime}\right)\right]=} & {\left[\omega_{\mathrm{CS}}^{(I)}(t) I_{z}+\omega_{\mathrm{CS}}^{(S)}(t) S_{z}-d_{I S}(t)\left[3 I_{z} S_{z}-I S\right],\right.} \\
& \left.\omega_{\mathrm{CS}}^{(D}\left(t^{\prime}\right) I_{z}+\omega_{\mathrm{CS}}^{(S)}\left(t^{\prime}\right) S_{z}-d_{I S}\left(t^{\prime}\right)\left[3 I_{z} S_{z}-I S\right]\right],
\end{aligned}
$$

where the frequency coefficients $\omega_{\mathrm{CS}}$ and $d_{I S}$ are anisotropic, although the former comprises an isotropic part. The commutator of eqn (7) vanishes if the spins $I$ and $S$ are equivalent, so that $\omega_{\mathrm{CS}}^{(I)}(t)=\omega_{\mathrm{CS}}^{(S)}(t)$ and $\omega_{\mathrm{CS}}^{(I)}\left(t^{\prime}\right)=\omega_{\mathrm{CS}}^{(S)}\left(t^{\prime}\right)$. As a result, the $2 \mathrm{D}$ amplified PASS experiment is expected to perform correctly in our case where the dipolar coupling is refocused by rotation about the magic angle, provided the observation is performed stroboscopically with the rotor period. This implies that, as in the previously described static $2 \mathrm{D}$ experiment, the resulting indirect $F_{1}$ dimension is again free of dipolar effects. Furthermore, as a result of the amplification effect $N$, the $F_{1}$ projection mirrors the intensities of the spinning sidebands that one would acquire if a simple dipolar echo experiment could be performed with a fictitious spinning rate $\nu_{\mathrm{Rot}}^{\prime}=\nu_{\mathrm{Rot}} / N$, i.e., if one could generate more spinning sidebands to improve the mapping of the anisotropy. The 2D-amplified PASS spectrum of $\mathrm{Ba}\left(\mathrm{ClO}_{3}\right)_{2} \cdot \mathrm{H}_{2} \mathrm{O}$ is shown in Fig. 4a. The spinning sideband pattern in $F_{2}$ is affected both by the dipolar and shift interactions, as in a simple dipolar echo experiment. In contrast, $F_{1}$ produces a series of peaks which mimic the spinning sideband intensities due to the shift interaction only. Moreover, having been recorded at $\nu_{\text {rot }}=3333 \mathrm{~Hz}$ with an amplification factor $N=$ 3.333 , the spinning sideband pattern presented in $F_{1}$ resembles that acquired at a fictitious spinning rate of $\nu_{\mathrm{rot}}^{\prime}=1 \mathrm{kHz}$. This yields a larger number of spinning sidebands to analyze. Fig. $4 \mathrm{~b}$ shows fitting of the $F_{1}$ projection calculated by summing $F_{1}$ slices extracted at each spinning sideband and numerical fit, in black and red, respectively. Again, in this spinning case, as in the static experiment of Fig. 3, only one single $I=1 / 2$ spin needs to be taken into account in the fit, without any need for simulating the entire multiple-pulse two-dimensional experiment. As in Fig. 3, the pure-shift $F_{1}$ dimension is independent of the relative orientation of the dipolar and shielding tensors. The agreement between the fit and the experiment is generally good. This is particularly evident when one considers the $2 \mathrm{D}$ plot of the rms deviations in Fig. 4c, which allows one to extract the relevant parameters $\Delta_{\mathrm{CS}}=-8.5 \pm 0.6 \mathrm{ppm}$ and $\eta_{\mathrm{CS}}=1 \pm 0.1$ with greatly improved accuracy and precision compared to those obtained from 1D spectra. As in the static spectrum of Fig. 3, we are able to measure accurately and fully characterize a relatively small chemical shift interaction of $c a .4 \mathrm{kHz}$ by removing the predominant dipolar interaction of $c a .30 \mathrm{kHz}$ under spinning conditions. Furthermore, the agreement with the averaged parameters calculated by DFT methods is good. The anisotropy $\Delta_{\mathrm{CS}}$ and asymmetry $\eta_{\mathrm{CS}}$ of the motionallyaveraged proton shielding tensor measured experimentally in this study are summarized in Table 2.

The contour plots resulting from both pure-shift 2D NMR techniques used in this study also carry information about the relative orientation between the shielding and dipolar tensors. This feature can be easily appreciated in Fig. 5, where numerical simulations of the Antonijevic-Wimperis and 2D-amplified PASS experiments are shown for the three cases of $\Omega_{\mathrm{PC}}^{\mathrm{D}}=$ $\left(0^{\circ}, 0^{\circ}, 0^{\circ}\right),\left(0^{\circ}, 45^{\circ}, 0^{\circ}\right)$ and $\left(0^{\circ}, 90^{\circ}, 0^{\circ}\right)$. These simulations clearly show that (i) the 2D correlation lineshapes depend on the relative orientation between the two interactions and (ii) the $F_{1}$ projections are instead independent with respect to this feature. Although a thorough analysis of the $2 \mathrm{D}$ correlation lineshapes has not been undertaken in this study, the comparison between our experimental evidence and numerical simulations performed with the SIMPSON code seems to suggest that the Euler angle which relates the main $z$-axes of the dipolar and shielding tensors is close to zero. This is not consistent with an averaging motion given by 2 -fold flips about the $\mathrm{H}-\mathrm{O}-\mathrm{H}$ bisector, which would instead yield $\beta=90^{\circ}$. Further studies may be required to

Table 2 Experimental values of anisotropy $\Delta_{\mathrm{CS}}$ and asymmetry $\eta_{\mathrm{CS}}$ as measured with 1D static and spinning solid echoes and 2D static and spinning pure-shift methods

\begin{tabular}{llrrr}
\hline NMR method & & $\beta$ & $\Delta_{\mathrm{CS}}(\mathrm{ppm})$ & \multicolumn{1}{c}{$\eta_{\mathrm{CS}}$} \\
\hline \multirow{2}{*}{ 1D solid echo } & Static & $0^{\circ}$ & $-10 \pm 3$ & $1 \pm 0.7$ \\
& & $90^{\circ}$ & $11 \pm 3$ & $0.3 \pm 0.5$ \\
& Spinning & $0^{\circ}$ & $-9 \pm 7$ & $0 \pm 11$ \\
& & $90^{\circ}$ & $9 \pm 10$ & $1 \pm 2$ \\
2D pure-shift & Static & - & $-10.5 \pm 1.0$ & $0.7 \pm 0.2$ \\
& Spinning (amplified) & - & $-8.5 \pm 0.6$ & $1 \pm 0.1$
\end{tabular}


a)

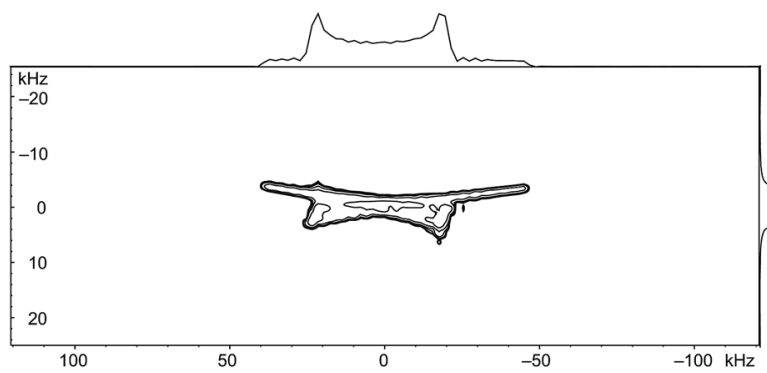

b)

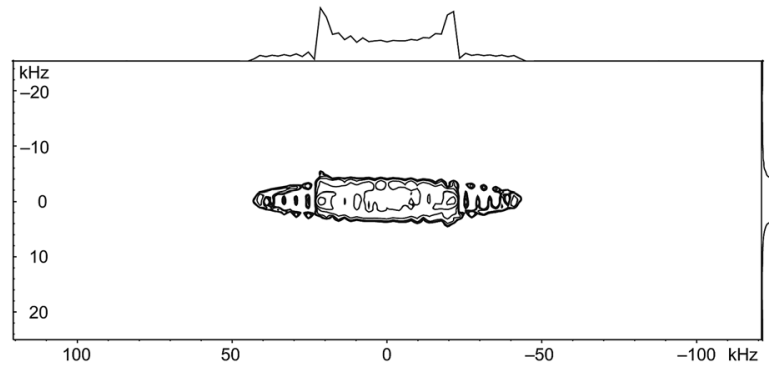

c)

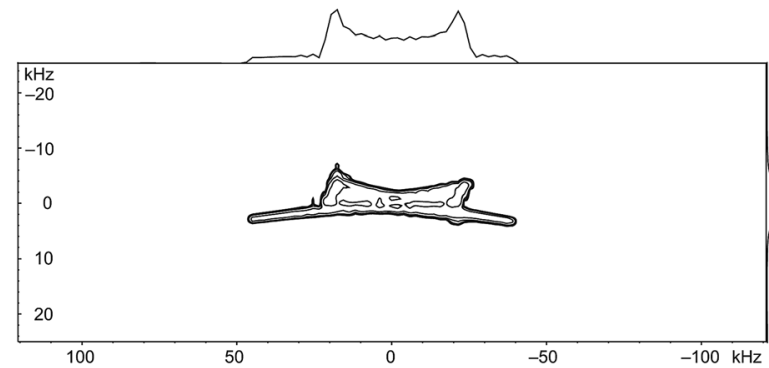

d)

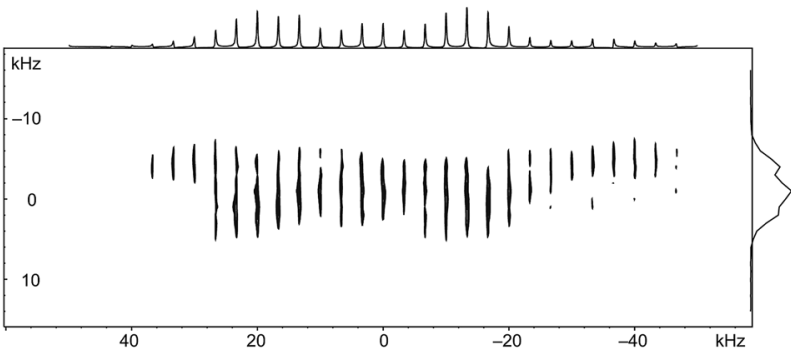

e)

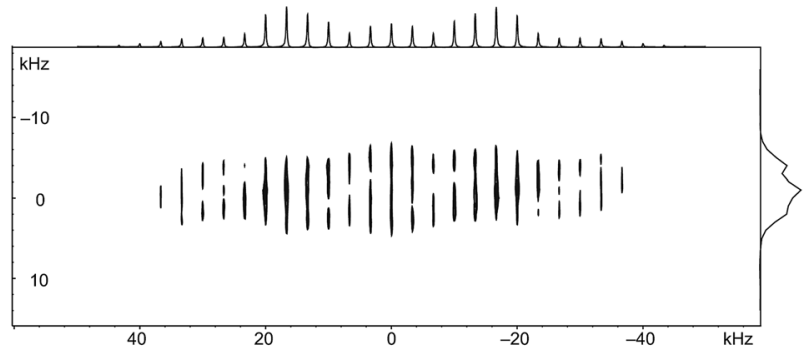

f)

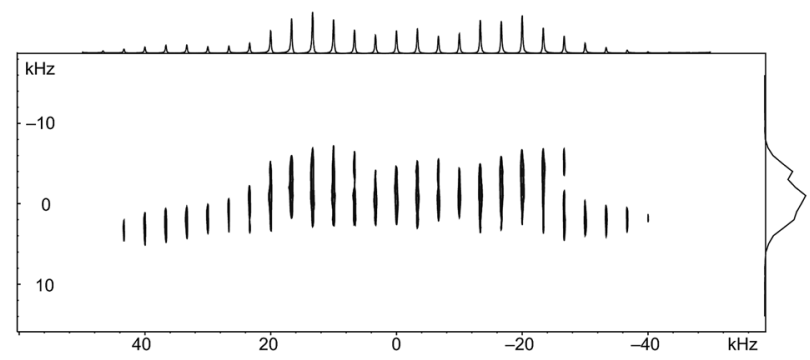

Fig. 5 Numerical simulations of the static 2D correlation experiment shown in Fig. 3a with $\beta=0,45$ and $90^{\circ}$, in (a), (b) and (c), respectively. Numerical simulations of the spinning 2D correlation experiment shown in Fig. 4 a with $\beta=0,45$ and $90^{\circ}$, in (d), (e) and (f), respectively. Realistic pulses have been assumed in all cases and coherence selection was taken into account. The isotropic shift was $\delta_{\text {iso }}=0 \mathrm{kHz}$ whereas the anisotropy $\Delta_{\mathrm{CS}}$ and asymmetry $\eta_{\mathrm{CS}}$ were those in Table 2 for the corresponding experiments. All simulations assumed an external magnetic field $B_{0}=9.4 \mathrm{~T}$ and a dipolar coupling constant $d=-29 \mathrm{kHz}$.

investigate and interpret this finding in terms of types of motion of water molecules in $\mathrm{Ba}\left(\mathrm{ClO}_{3}\right)_{2} \cdot \mathrm{H}_{2} \mathrm{O}$.

\section{Conclusions}

The chemical shift tensors of the protons of isolated water molecules trapped in barium chlorate monohydrate $\mathrm{Ba}\left(\mathrm{ClO}_{3}\right)_{2} \cdot \mathrm{H}_{2} \mathrm{O}$ have been characterized by solid-state NMR under both spinning and static conditions. Two-dimensional techniques allow the measurement of the chemical shift anisotropy by selectively refocusing the predominant dipolar interaction from the indirect dimension. This produces a pure-shift indirect dimension in the resulting 2D spectra from which the main components of the interaction tensors can be easily determined. By means of these techniques one can greatly reduce the number of parameters to consider when analyzing the lineshapes. In particular, the relative orientation between the shielding and dipolar tensors can be neglected. The measured values of the anisotropy and asymmetry agree well with the motionallyaveraged values calculated for a periodic system with planewave pseudopotential DFT methods, as implemented in the CASTEP code.

\section{Experimental and computational details}

$\mathrm{Ba}\left(\mathrm{ClO}_{3}\right)_{2} \cdot \mathrm{H}_{2} \mathrm{O}$ was purchased from Alfa Aesar and kept under vacuum overnight to minimize the adsorption of water which can give rise to a misleading sharp isotropic peak in the middle of the Pake pattern. All $1 \mathrm{D}{ }^{1} \mathrm{H}$ MAS and 2D static spectra were recorded at $T=300 \mathrm{~K}$ on a Bruker 400 spectrometer (9.4 T) with an Avance-II console, using $3.2 \mathrm{~mm}$ rotors in a triple-resonance probe designed for low-temperature DNP measurements. The rf-field amplitude was $\nu_{1}=125 \mathrm{kHz}$, corresponding to $90^{\circ}$ pulses of length $\tau_{p}=2 \mu \mathrm{s}$. The $2 \mathrm{D}$ spinning spectrum was recorded at room temperature on a Bruker 600 spectrometer (14.1 T) equipped with an AVANCE-III console and a widebore $4 \mathrm{~mm}$ probe. The rf-field amplitude was $\nu_{1}=100 \mathrm{kHz}$, corresponding to $90^{\circ}$ pulses with $\tau_{p}=2.5 \mu \mathrm{s}$. The proton chemical shifts were referenced to adamantane at $1.8 \mathrm{ppm}$. Spinning frequencies of $\nu_{\text {rot }}=3.333$ or $10 \mathrm{kHz}$ were used. Typical recycle intervals varied between 3 and $20 \mathrm{~s}$. All $1 \mathrm{D} 90^{\circ}-\tau-90^{\circ}-\tau$ solid echoes were phasecycled for the selection of the $p=0 \rightarrow p=+1 \rightarrow p=-1$ pathway by nesting Exorcycle ${ }^{34}$ with Cyclops ${ }^{35}$ so to produce an overall 16-step phase cycle. ${ }^{18}$ The refocusing delay was $\tau=50 \mu \mathrm{s}$ and $100 \mu \mathrm{s}$ (for $\nu_{\text {rot }} 10 \mathrm{kHz}$ ) for static and spinning 1D spectra, 
respectively. The $2 \mathrm{D}$ static pure-shift spectrum of Fig. 3a was acquired by averaging 256 transients for each of $50 t_{1}$ increments of $10 \mu \mathrm{s}, \tau=180 \mu \mathrm{s}$ and a recycling delay of $25 \mathrm{~s}$. The $2 \mathrm{D}-$ amplified PASS experiment of Fig. 4a resulted from the averaging of 14 transients for each of $32 t_{1}$ increments, a spinning rate $\nu_{\text {rot }}=3.333 \mathrm{kHz}$, an amplification factor $N=3.333$ and a recycle delay of $3 \mathrm{~s}$. For this latter experiment, cogwheel phase cycling $^{36}$ was used to minimize the number of transients required. Numerical simulations and fits were performed with SIMPSON, ${ }^{37}$ using 2000 crystal orientations sampled with the REPULSION scheme ${ }^{38}$ for static spectra, whereas 323 orientations were sampled with the CZW scheme ${ }^{39-41}$ over $12 \gamma$-angles for spinning spectra. The rms plotted in Fig. $3 \mathrm{c}$ and $4 \mathrm{c}$ is provided as output by SIMPSON, as described in the manual.

Calculations of total energies and NMR parameters were carried out using the CASTEP DFT code (version 6), ${ }^{22}$ employing the gauge-including projector augmented wave (GIPAW) ${ }^{\mathbf{4 2}}$ algorithm to reconstruct the all-electron wave function in the presence of a magnetic field. Calculations were performed using the GGA PBE functional ${ }^{43}$ and core-valence interactions were described by ultrasoft pseudopotentials. ${ }^{\mathbf{4 4}}$ A planewave energy cutoff of 60 Ry was used, and integrals over the Brillouin zone were performed using a $k$-point spacing of $0.04 \AA^{-1}$. All calculations were allowed to converge as far as possible with respect to both $k$-point spacing and cutoff energy. Calculations were performed on a 198-node (2376 core) Intel Westmere cluster with 2 GB memory per core and QDR Infiniband interconnect at the University of St Andrews. The reduced shielding anisotropy $\Delta_{\mathrm{CS}}$ as used in this work is obtained by multiplying the full shielding tensor as calculated with CASTEP by the factor $2 / 3$.

\section{Abbreviations}

$\begin{array}{ll}\text { MAS } & \text { Magic-angle spinning } \\ \text { CSA } & \text { Chemical shift anisotropy } \\ \text { rms } & \text { Root-mean square } \\ \text { DFT } & \text { Density functional theory }\end{array}$

\section{Acknowledgements}

The authors would like to thank Martial Rey and Dr Daniel Dawson for technical assistance and Prof Malcolm H. Levitt, Dr Robin Orr, Dr John M. Griffin, Dr Piotr Tekely and Dr Daniel Abergel for constructive discussions. This work was supported by the Swiss National Science Foundation (SNSF), the Ecole Polytechnique Fédérale de Lausanne (EPFL), the Swiss Commission for Technology and Innovation (CTI) and the European Research Council (ERC, contract 'dilute para-water').

\section{References}

1 G. Hura, D. Russo, R. M. Glaeser, T. Head-Gordon, M. Krack and M. Parrinello, Phys. Chem. Chem. Phys., 2003, 5, 1981.

2 P. Postorino, R. H. Tromp, M.-A. Ricci, A. K. Soper and G. W. Neilson, Nature, 1993, 366, 668.
3 H. E. Stanley and J. Texeira, J. Chem. Phys., 1980, 73(7), 3404.

4 C. R. Bowers and D. P. Weitekamp, J. Am. Chem. Soc., 1987, 109, 5541.

5 H. Eisendarth, W. Stone and J. Jeener, Phys. Rev. B: Condens. Matter Mater. Phys., 1978, 17, 47.

6 T. Kravchuk, M. Reznikov, P. Tichonov, N. Avidor, Y. Meir, A. Bekkerman and G. Alexandrowicz, Science, 2011, 331, 319.

7 C. Beduz, M. Carravetta, J. Y.-C. Chen, M. Concistrè, M. Denning, M. Frunzi, A. J. Horsewill, O. G. Johannessen, R. Lawler, X. Lei, M. H. Levitt, Y. Li, S. Mamone, Y. Murata, U. Nagel, T. Nishida, J. Ollivier, S. Rols, T. Rõom, R. Sarkar, N. J. Turro and Y. Yang, Proc. Natl. Acad. Sci. U. S. A., 2012, 109(32), 12894.

8 M. J. Duer, Solid-State NMR Spectroscopy: Principles and Applications, Blackwell Science, UK, 2002.

9 E. R. Andrew, A. Bradbury and R. G. Eades, Nature, 1958, 182, 1659.

10 I. Lowe, Phys. Rev. Lett., 1959, 22, 133.

11 C. Bonhomme, C. Gervais, F. Babonneau, C. Coelho, F. Pourpoint, T. Azaïs, S. E. Ashbrook, J. M. Griffin, J. R. Yates, F. Mauri and C. J. Pickard, Chem. Rev., 2012, 112, 5733.

12 T. Charpentier, Solid State Nucl. Magn. Reson., 2011, 40(1), 1. 13 M. R. Mitchell, D. Carnevale, R. Orr, K. R. Whittle and S. E. Ashbrook, J. Phys. Chem. C, 2012, 116, 4273.

14 M. Castro, V. R. Seymour, D. Carnevale, J. M. Griffin, S. E. Ashbrook, P. A. Wright, D. C. Apperley, J. E. Parker, S. P. Thompson, A. Fecant and N. Bats, J. Phys. Chem. C, 2010, 114, 12698.

15 D. Carnevale, V. del Amo, D. Philp and S. E. Ashbrook, Tetrahedron, 2010, 66, 6238.

16 K. Modig and B. Halle, J. Am. Chem. Soc., 2002, 124, 12031.

17 P. Tekely, P. Palmas and P. Mutzenhardt, J. Magn. Reson., 1997, 127, 238.

18 S. Antonijevic and S. Wimperis, J. Magn. Reson., 2003, 164, 343.

19 G. E. Pake, J. Chem. Phys., 1948, 16(4), 327.

20 J. R. Long, R. Ebelhäuser and R. G. Griffin, J. Phys. Chem. A, 1997, 101, 988.

21 S. Antonijevic and S. Wimperis, J. Chem. Phys., 2005, 122, 044312.

22 M. D. Segall, P. J. D. Lindan, M. J. Probert, C. J. Pickard, P. J. Hasnip, S. J. Clark and M. C. Payne, J. Phys.: Condens. Matter, 2002, 14, 2717.

23 S. K. Sikka, S. N. Momin, H. Rajagopal and R. Chidambaram, J. Chem. Phys., 1968, 48(5), 1883.

24 S. P. Brown and S. Wimperis, J. Magn. Reson., 1997, 124, 279.

25 R. M. Orr, M. J. Duer and S. E. Ashbrook, J. Magn. Reson., 2005, 174, 301.

26 R. M. Orr and M. J. Duer, Solid State Nucl. Magn. Reson., 2006, 30, 1.

27 C. Crockford, H. Geen and J. J. Titman, Chem. Phys. Lett., 2001, 344, 367.

28 L. Shao, C. Crockford, H. Geen, G. Grasso and J. J. Titman, J. Magn. Reson., 2004, 167, 75.

29 O. N. Antzutkin, S. C. Shekar and M. H. Levitt, J. Magn. Reson., 1995, 115, 7. 
30 W. T. Dixon, J. Magn. Reson., 1981, 44, 220.

31 W. T. Dixon, J. Chem. Phys., 1982, 77, 1800.

32 D. Haeberlen and J. S. Waugh, Phys. Rev., 1968, 175, 453.

33 W. Magnus, Comm. Pure Appl. Math., 1954, 7, 649.

34 G. Bodenhausen, R. Freeman and D. L. Turner, J. Magn. Reson., 1977, 27, 511.

35 D. I. Hoult and R. E. Richards, Proc. - R. Soc. Edinburgh, Sect. A: Math., 1975, 344, 311.

36 M. H. Levitt, P. K. Madhu and C. E. Hughes, J. Magn. Reson., 2002, 155, 300.

37 M. Bak, J. T. Rasmussen and N. C. Nielsen, J. Magn. Reson., 2000, 147, 296.
38 M. Bak and N. C. Nielsen, J. Magn. Reson., 1997, 125, 132.

39 S. K. Zaremba, Ann. Mat. Pura Appl., 1966, 73(1), 293-317.

40 H. Conroy, J. Chem. Phys., 1967, 47, 5307.

41 V. B. Cheng, H. H. Suzukawa Jr and M. Wolfsberg, J. Chem. Phys., 1973, 59, 3992.

42 C. J. Pickard and F. Mauri, Phys. Rev. B: Condens. Matter Mater. Phys., 2001, 63, 245101.

43 J. P. Perdew, K. Burke and M. Ernzerhof, Phys. Rev. Lett., 1996, 77, 3865.

44 J. R. Yates, C. J. Pickard and F. Mauri, Phys. Rev. B: Condens. Matter Mater. Phys., 2007, 76, 024401. 